\title{
The hardness evolution of gamma-ray burst pulses
}

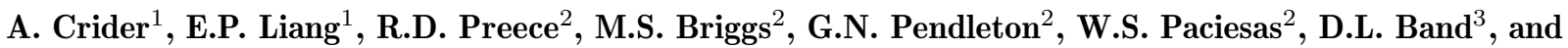 \\ J.L. Matteson ${ }^{3}$ \\ 1 Department of Space Physics and Astronomy, 6100 S. Main, Rice University, Houston, TX 77005-1892, U.S.A. \\ 2 Department of Physics, University of Alabama in Huntsville, Huntsville, AL 35899, U.S.A. \\ 3 Center for Astrophysics and Space Sciences 0424, University of California at San Diego, La Jolla, CA 92093, U.S.A.
}

Received January 21; accepted April 21, 1999

\begin{abstract}
We have analyzed the evolution of the spectral hardness parameter, $E_{\mathrm{pk}}$ (the maximum of the $\nu F_{\nu}$ spectrum) as a function of fluence in gamma-ray bursts. We fit 41 pulses within 26 bursts with the trend reported by Liang \& Kargatis (1996) which found that $E_{\mathrm{pk}}$ decays exponentially with respect to photon fluence $\Phi(t)$. We also fit these pulses with a slight modification of this trend, where $E_{\mathrm{pk}}$ decays linearly with energy fluence. In both cases, we found the set of 41 pulses to be consistent with the trend.
\end{abstract}

Key words: gamma-ray: bursts, observations

\section{Introduction}

The observed fading multi-wavelength afterglows of gamma-ray bursts are so far consistent with the simple relativistic blastwave model (Mészáros \& Rees 1997). The emission mechanism resulting in the prompt gamma rays remains a mystery. Studies of gamma-ray burst (GRB) spectral evolution have uncovered many trends which may be used to confront models. The discovery that peak power energy $E_{\mathrm{pk}}$ (which is the maximum of $\nu F_{\nu}$, where $\nu$ is photon energy and $F_{\nu}$ is the specific energy flux) often decays exponentially in bright, long, smooth BATSE GRB pulses as a function of photon fluence $\Phi$ $\left(=\int_{t^{\prime}=0}^{t^{\prime}=t} F_{\mathrm{N}}\left(t^{\prime}\right) \mathrm{d} t^{\prime}\right)$ (Liang \& Kargatis 1996, hereafter LK96) provided a new constraint on emission mechanisms (Liang et al. 1997; Liang 1997; Daigne \& Mochkovitch 1998). In their analysis, LK96 fit the function

$E_{\mathrm{pk}}(t)=E_{\mathrm{pk}(0)} \mathrm{e}^{-\Phi(t) / \Phi_{0}^{\mathrm{LK}}}$

to 37 GRB pulses in 34 bursts. To interpret this empirical trend, they differentiated Eq. (1) to find

$-\mathrm{d} E_{\mathrm{pk}} / \mathrm{d} t=E_{\mathrm{pk}} F_{\mathrm{N}} / \Phi_{0}^{\mathrm{LK}} \approx F_{\mathrm{E}} / \Phi_{0}^{\mathrm{LK}}$

Send offprint requests to: A. Crider where $F_{\mathrm{E}}=\int_{\mathrm{E} \approx 30 \mathrm{keV}}^{E \approx 2000 \mathrm{keV}} E N(E) \mathrm{d} E$ is the BATSE energy flux and $F_{\mathrm{N}}=\int_{E \approx 30 \mathrm{keV}}^{E \approx 2000 \mathrm{keV}} N(E) \mathrm{d} E$ is the BATSE photon flux (see Eq. (1) of LK96). We wished to avoid the assumption that $E_{\mathrm{pk}} F_{\mathrm{N}} \approx F_{\mathrm{E}}$ and instead directly tested the trend $-\mathrm{d}\left(E_{\mathrm{pk}}\right) / \mathrm{d} t=F_{\mathrm{E}} / \Phi_{0}$ by integrating it to give

$E_{\mathrm{pk}}(t)=E_{\mathrm{pk}(0)}-\mathcal{E}(\mathrm{t}) / \Phi_{0}$

where $\mathcal{E}(\mathrm{t})\left(=\int_{t^{\prime}=0}^{t^{\prime}=t} F_{\mathrm{E}}\left(t^{\prime}\right) \mathrm{d} t^{\prime}\right)$ is the BATSE energy fluence. Our motivation for using the $E_{\mathrm{pk}}$-energy fluence relation (Eq. (3)) as opposed the $E_{\mathrm{pk}}$-photon fluence relation (Eq. (1)) is that we believe that the former represents a more physical quantity. The BATSE LAD energy window was designed to contain the peak of GRB energy spectra, not the peak of the photon spectra. By using energy fluence, we avoid the somewhat shaky assumption that the BATSE LAD photon flux is proportional to the bolometric photon flux.

\section{Procedures}

To determine the evolution of GRB spectral shapes, we examined High Energy Resolution data collected from the BATSE Large-Area Detectors (LADs) and Spectroscopy Detectors (SDs) We began with the 126 bursts from the catalog developed for Preece et al. (1998). We deconvolved the gamma-ray spectra of each time interval using the Band et al. (1993) GRB function. While LK96 assumed that the Band GRB parameters $\alpha$ and $\beta$ were constant during the course of each burst, this has since been shown to be untrue with a larger data set (Crider et al. 1997). We thus left $\alpha$ and $\beta$ as free parameters in our fits.

At this point, we needed to select pulses within our bursts that we could use to test Eq. (1). Unfortunately, by forcing our time bins to have a SNR $\sim 45$ so that spectra may be fit to them, much time resolution is lost. Pulses which would be easily separable at a higher time resolution became blurred together. In Fig. 1, we show an example of what would likely be identified as two pulses in our 

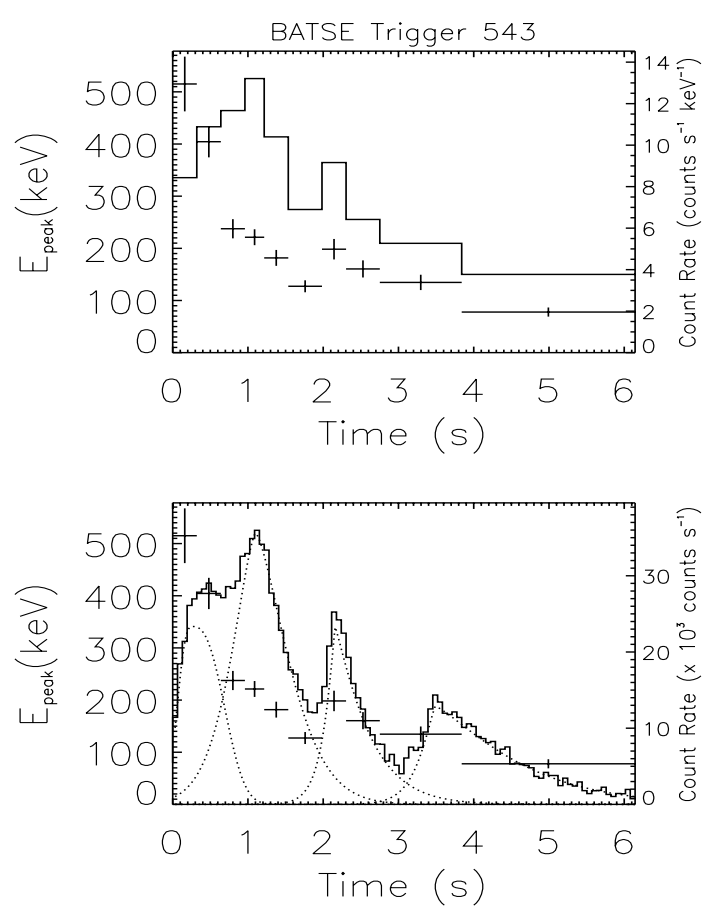

Fig. 1. The count rate (plotted as a histogram) of BATSE trigger 543 seen both in a) the lower time resolution which allowed us to fit a spectrum to each bin and b) 64-ms resolution. $E_{\mathrm{pk}}$, as determined from the data in the upper plot, is marked on both plots for convenience. The fits of the Norris function to these pulses appear as dotted lines

coarse data. Below it, we plot 64 -ms count rate data for this same burst, obtained from the Compton Observatory Science Support Center (COSSC). With higher time resolution, we see this burst is composed of at least 4 distinct pulses. We used the COSSC 64-ms data to determine where each of our pulses began and ended by developing an interactive IDL routine to fit the Norris et al. (1996) pulse profile to the individual pulses within our bursts. We found that in many bursts, pulses overlapped in a fashion making them too complex for us to fit individual pulses. Other bursts contained pulses which could be resolved, but none of their pulses lasted long enough to span at least 4 time bins with SNR $\sim 45$. For 13 of the bursts, no processed 64 -ms data was available. Discarding bursts which fell into any of these three categories, left us with 26 bursts. Within these, we identified 41 regions composed of at least 4 time bins dominated by a single pulse (at least $70 \%$ of the counts from one pulse).

\section{Results}

We fit the $E_{\mathrm{pk}}-\mathcal{E}$ relation to our 41 clean pulses using FITEXY (Press et al. 1992). From the $\chi^{2}$ and the number of fluence bins for each decay fit, we calculated the probability $Q$ of randomly getting a higher $\chi^{2}$ by chance. If $E_{\mathrm{pk}}$ does indeed cool linearly with $\mathcal{E}$ in all pulses selected for fitting, then when plotting the cumulative distribution

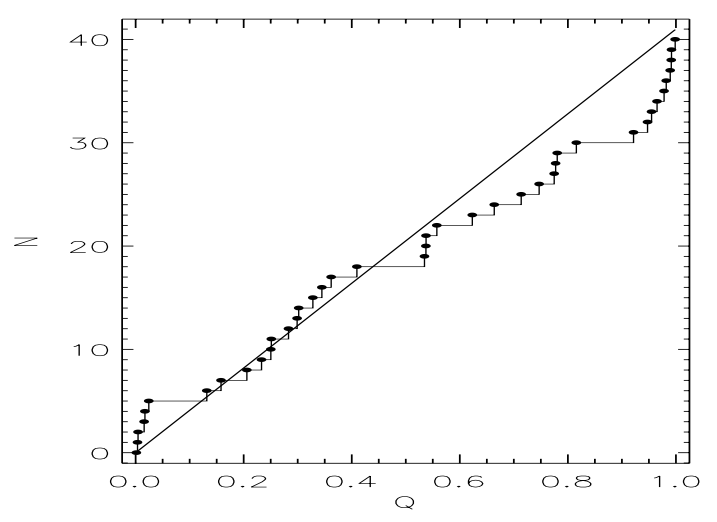

Fig. 2. Cumulative distribution of $Q$ values for 41 pulses. In this plot, $N$ is the fraction of pulses that have a $Q$ value less than that of a certain pulse. If all of these GRB pulses cooled linearly as a function of energy fluence, one would expect the cumulative $Q$ distribution to match the unit distribution $(N=Q)$

of $Q$ values, we would expect $10 \%$ of the pulses to have a $Q$ less that $0.1,20 \%$ of the pulses to have a $Q$ less than 0.2 , and so on. Figure 2 shows the cumulative distribution of $Q$ values for our pulses with acceptable fits. An excess of pulses with very high $Q$ values would suggest a biased pulse selection process. The Kolmogorov-Smirnov test gives a probability $P=0.18$ that this distribution is drawn from the unit distribution. From this, we conclude that our $E_{\mathrm{pk}}-\mathcal{E}$ function (Eq. (3)) adequately describes the pulses in this subset. We discuss this work and its implications in more detail elsewhere (Crider et al. 1999).

Acknowledgements. AC thanks NASA-MSFC for his GSRP fellowship and the Rome GRB Conference Committee for waiving his conference fees. AC also wishes to acknowledge useful discussions with Charles Dermer and Andrew Lemanski that greatly influenced this paper. This work is supported by NASA grant NAG5-3824.

\section{References}

Band D., et al., 1993, ApJ 413, 281

Crider A., et al., 1999, ApJ 519, 206-213

Crider A., et al., 1997, ApJ 479, L39

Daigne F., Mochkovitch R., 1998, MNRAS 296, Iss. 2, 275

Liang E.P., Kargatis V.E., 1996, Nat 381, 49

Liang E.P., Smith I.A., Kusunose M., Crider A., 1997, ApJ 479, L35

Liang E.P., 1997, ApJ 491, L15

Mészáros P., Rees M.J., 1997, ApJ 476, 232

Norris J.P., et al., 1996, ApJ 459, 393

Preece R.D., et al., 1998, ApJ 496, 849

Press W.H., Teukolsky S.A, Vettering W.T, Flannery B.P., Numerical Recipes in C, 2nd Edition, 1992. Cambridge: Cambridge University 